\title{
ESTUDIO MORFOMÉTRICO DE LA BIFURCACIÓN ILÍACA Y SUS IMPLICANCIAS AL USO DE DISPOSITIVOS CON RAMA ILÍACA
}

\author{
Leticia Vázquez, María F. Ignatov, Juan E. Kenny, Alejandro M. Russo \\ Departamento de Anatomía, Facultad de Medicina, Universidad de la República, \\ Montevideo, Uruguay
}

\section{RESUMEN}

Introducción: La reparación endovascular de los aneurismas aorto-ilíacos con dispositivos de rama ilíaca permiten excluir el aneurisma de forma eficaz, así como mantener la circulación de la pelvis. Sin embargo, no todas las anatomías son aptas para esta tecnología. Esto motivó el siguiente estudio, cuyo objetivo es evaluar la compatibilidad de la anatomía de los vasos ilíacos con respecto a los valores preestablecidos de estos dispositivos, en una población cadavérica. Materiales y métodos: Se disecaron 53 bifurcaciones ilíacas arteriales, de cadáveres adultos formolados. Se registró: sexo, lado (derecho o izquierdo), longitud de la arteria ilíaca común (L1), longitud de la arteria iliaca interna (L2), y calibres de la arteria ilíaca común (C1), de la bifurcación ilíaca (C2), de la arteria iliaca interna (C3) y externa (C4). Las variables cuantitativas fueron comparadas con los requerimientos de 3 casas comerciales que fabrican estos dispositivos. Resultados: Los valores promedio obtenidos fueron de: L1: $54,52 \mathrm{~mm}$; L2: $40,59 \mathrm{~mm}$; C1: $11,73 \mathrm{~mm}$; C2: 15,34 mm; C3: 7,28 mm; C4: $8,36 \mathrm{~mm}$. Del total de casos estudiados, 5 casos $(9.43 \%)$ cumplieron con los requisitos de la primera casa comercial, los restantes $48(90,57 \%)$ no lo hicieron. Ocho casos $(15,09 \%)$ cumplieron con los de la segunda casa, 45 (84,91\%) no lo hicieron. Finalmente, 18 casos $(33,96 \%)$ cumplieron con los requisitos de la tercer casa comercial, mientras que los restantes 35 casos $(66,04 \%)$ no lo hicieron. Conclusiones: El cirujano debe estar al tanto de esta y otras técnicas de sello distal de aneurismas aorto-ilíacos, ya que no todas las bifurcaciones iliacas cumplen los requisitos de las casas comerciales. Es fundamental determinar la morfometría de cada caso para ver su compatibilidad con los dispositivos.

Palabras clave: Aorta. Arteria ilíaca común. Arteria iliaca interna. Aneurisma. Anatomía Clínica.

\begin{abstract}
Introduction: Endovascular repair of aorto-iliac aneurysms by means of iliac branch devices allows both proper aneurysmal sealing and patency of the pelvic circulation. However, not all anatomies are suitable for this type of technology. This motivated the present study whose objective was to evaluate the compatibility of the anatomy of the iliac vessels to the specifications of the manufacturers of these devices, in a cadaveric population. Materials and methods: 53 arterial iliac bifurcations were dissected from adult formalin-fixed cadavers. It was registered: gender, side (right or left), length of the common iliac artery (L1), hypogastric arterial length (L2), and caliber of the common iliac artery (C1), iliac bifurcation (C2), hypogastric artery (C3) and external iliac artery (C4). These results were compared with the specifications of 3 manufacturers of iliac branch devices commonly used in our region. Results: The average values obtained were: L1: $54,52 \mathrm{~mm}$; L2: $40,59 \mathrm{~mm}$; C1: $11,73 \mathrm{~mm}$; C2: 15,34 mm; C3: 7,28 mm; C4: 8,36 mm. From the total cases studied, only 5 cases $(9.43 \%)$ met the requirements of the first manufacturer, the remaining 48 cases $(90,57 \%)$ did not. Eight (15,09\%) cases met the criteria of the second manufacturer while 45 specimens $(84,91 \%)$ did not. Eighteen cases $(33,96 \%)$ met the requirements of the third manufacturer while the remaining 35 cases $(66,04 \%)$ did not. Conclusions: Since not every case was suitable for iliac branch device, the surgeon must be aware of this and other type of devices for proper aortic-iliac aneurysmal distal sealing. It is crucial to determine the morphometry of each case in order to evaluate its suitability for iliac branch device use.
\end{abstract}

Keywords: Aorta. Common iliac artery. Internal Iliac artery. Aneurysm. Clinical Anatomy.

$\begin{array}{llll}* \quad \text { Correspondencia } & \text { a: Leticia Vázquez. } \\ \text { leticiavazquez@outlook.com } & \end{array}$

Recibido: 7 de Mayo, 2020. Revisado: 5 de Junio, 2020. Aceptado: 16 de Junio, 2020. 


\section{INTRODUCCIÓN}

La reparación endovascular de los aneurismas (EVAR) presenta ciertas ventajas respecto a la reparación convencional. Estas son: menor morbilidad y mortalidad del procedimiento, disminuye significativamente la pérdida sanguínea, el tiempo del procedimiento quirúrgico y la estancia hospitalaria. Adicionalmente, plantea una opción terapéutica para los pacientes cuyo riesgo anestésico quirúrgico es muy elevado (Oderich y Greenberg, 2011; Pearce et al, 2015). $\mathrm{Si}$ bien la presencia aislada de aneurismas de arteria ilíaca común es infrecuente (2\%) (Armon et al, 1998), la asociación de aneurismas de arteria aorta abdominal con aneurismas de arteria ilíaca es frecuente. La literatura reporta desde el 20\% (Armon et al, 1998; Pearce et al, 2015) hasta el $40 \%$ de asociación (Oderich y Greenberg, 2011; Jongsma et al, 2017). Por tanto, habitualmente se trata el aneurisma aórtico e ilíaco en el mismo acto operatorio. Hoy en día existen múltiples opciones terapéuticas para el tratamiento del aneurisma ilíaco. Se incluyen la reparación convencional, embolización de la arteria ilíaca interna, técnica en campana (del inglés "bell bottom technique"), colocación de stents en paralelo o el implante de un dispositivo con rama ilíaca (DRI). (Greenhalgh, 2018) (Figura 1).
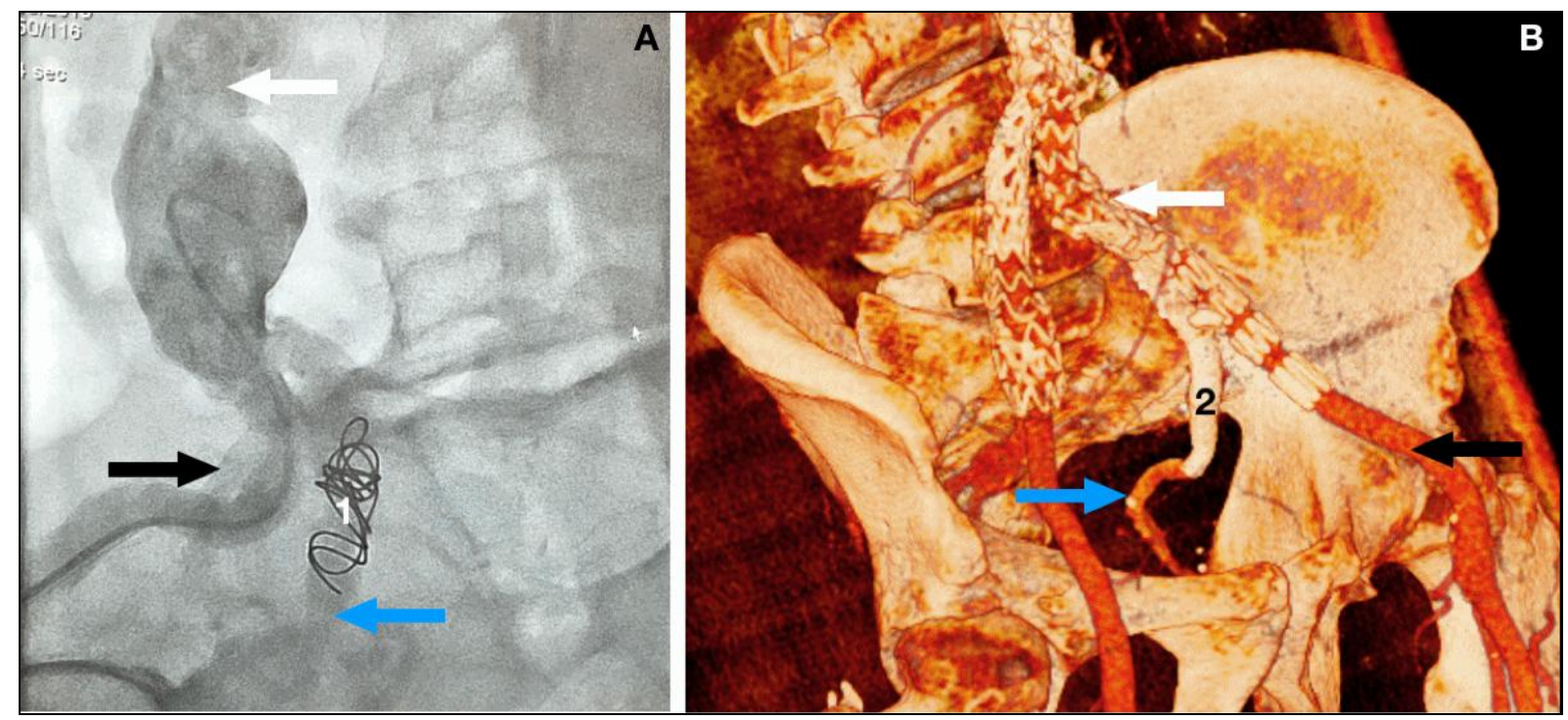

Figura 1- Imágenes de distintas formas de extender el sello distal en pacientes con aneurismas aorto-ilíacos. Imagenes del autor (AR). En A. angiografía post colocación de coil (1) en arteria ilíaca interna derecha (flecha azul). Flecha blanca arteria ilíaca común aneurismática. Flecha negra arteria ilíaca externa. En B. angiotomografía de control post implante de DRI. se observa el DRI (2) con arteria ilíaca interna distal permeable (flecha azul). Flecha blanca arteria ilíaca común, flecha negra arteria ilíaca externa.

Es práctica común extender el sello distal en dirección a la arteria ilíaca externa en pacientes con aneurisma ilíaco común. Esto último conlleva la obturación del ostium de la arteria ilíaca interna que previamente era ocluida ya sea mediante coils y/o oclusor, a modo de prevenir la endofuga tipo II (Greenhalgh, 2018) (Figura 1). Esta maniobra no está desprovista de complicaciones ya que se produce una alteración de la circulación pelviana. Estas complicaciones incluyen: claudicación glútea, impotencia sexual, isquemia colónica e isquemia de la médula espinal. La severidad de la isquemia pelviana está relacionada con la presencia y cantidad de la circulación colateral; sin embargo, los predictores clínicos y anatómicos de isquemia pélvica son difíciles de determinar de acuerdo a diversos investigadores (Oderich y Greenberg, 2011; D'oria et al, 2019).

La literatura ha reportado variabilidad en la incidencia de estas complicaciones, desde 13\% de claudicación glútea y ninguna otra complicación mayor (Criado et al, 2000) hasta 42 a $55 \%$ de claudicación glútea (Rayt et al, 2008; Pearce et al, 2015) junto con complicaciones mayores tales como disfunción eréctil de 10 a $46 \%$ (Jongsma et al, 2017; Kayssi y Neville, 2018; D'oria et al, 2019), isquemia colónica de $3,4 \%$ (Kayssi y Neville, 2018) e isquemia de médula espinal de 0,1 a $0,3 \%$ (Pearce et al, 
2015). Más allá de estos resultados, estudios observacionales han demostrado que la oclusión unilateral de la arteria ilíaca interna con la contralateral permeable se asocia a un bajo índice de complicaciones y sigue siendo la recomendación actual cuando se opta por su oclusión (Chaikof et al, 2018).

Para preservar la indemnidad de la circulación iliaca interna, y como técnica alternativa a este procedimiento, surgen los DRI. Los mismos respetan la anatomía del paciente y mantienen permeable la arteria iliaca interna descendiendo así los porcentajes de complicaciones antes descritos; a la vez que aseguran un adecuado sello del aneurisma (Schneider et al, 2017). Sin embargo, no todas las anatomías son aptas para este tipo de tecnología. Determinados elementos de la anatomía del paciente son criterios excluyentes para el uso de los DRI: tortuosidad de alguno o todos los vasos involucrados, trombos circunferenciales y calcificación o placas de ateroma en los sitios de fijación de la prótesis. El otro elemento que limita el uso de estos dispositivos es la morfometría de las arterias ilíacas en cuanto a longitud y calibre, en comparación a las medidas estipuladas por las instrucciones de uso (IFU) de las casas comerciales (Itoga et al, 2017).

Lo anteriormente expuesto, motivó el siguiente estudio cuyo objetivo es evaluar la compatibilidad de la anatomía iliaca en una población cadavérica con respecto a los requerimientos anatómicos en las IFU de los DRI más utilizados en nuestro medio.

\section{MATERIALES Y MÉTODOS}

Se disecaron 53 bifurcaciones ilíacas arteriales, correspondientes a 21 troncos y 11 hemi-pelvis (25 bifurcaciones ilíacas derechas y 28 izquierdas). Estas eran procedentes de cadáveres adultos, de ambos sexos (34 pelvis femeninas y 19 masculinas), previamente fijados y conservados en solución a base de formaldehido al $10 \%$. Se realizó el abordaje sucesivo de los planos de la pared abdominal, hasta acceder al retroperitoneo y realizar entonces una cuidadosa disección de los vasos ilíacos. (Figura 2).

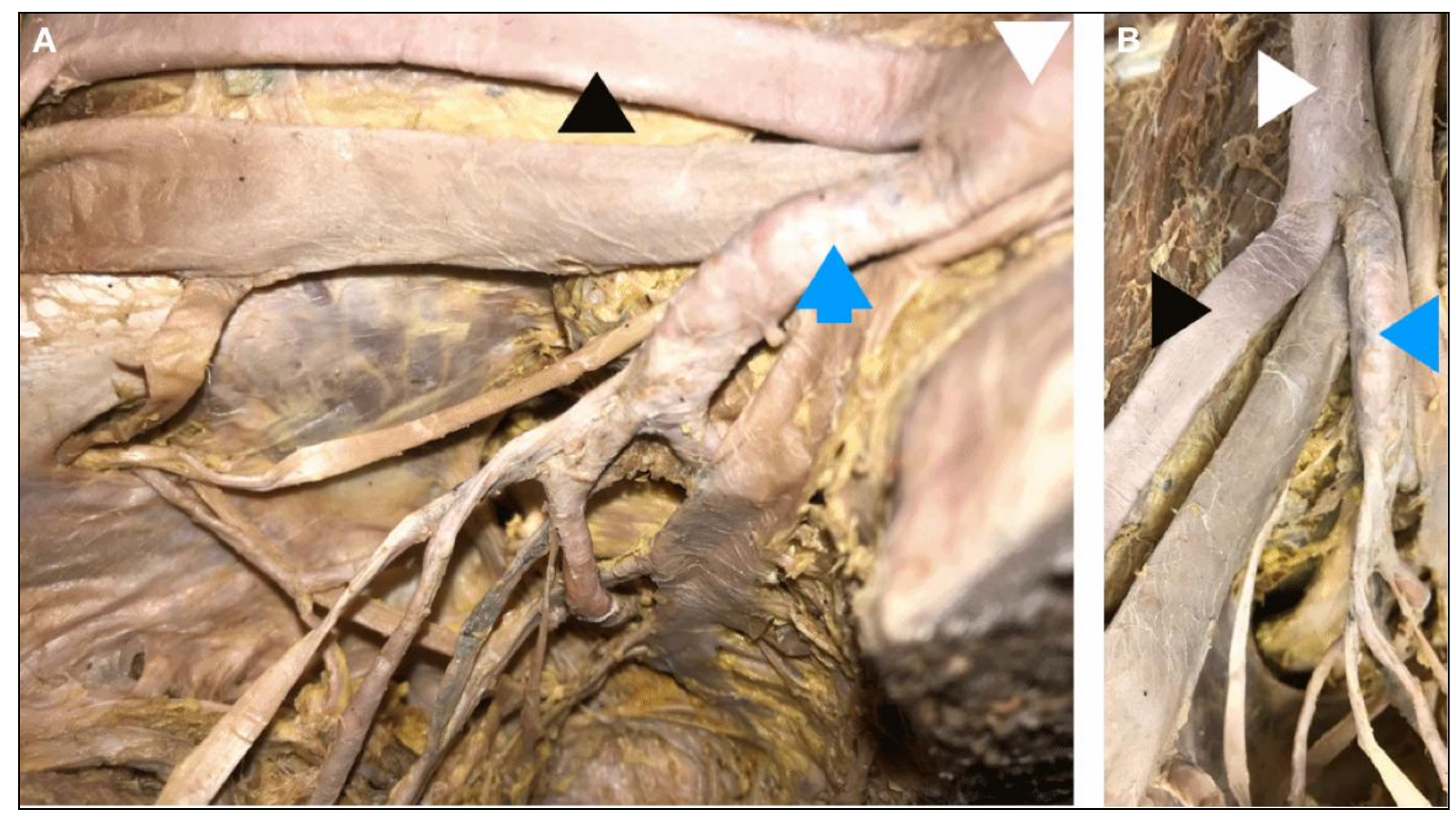

Figura 2- Imágenes de preparados cadavéricos fijados en formaldehído. En A. vista sagital de preparado. En B. vista superior del preparado. En ambas imágenes las flechas indican lo siguiente: flecha blanca arteria ilíaca común, flecha negra arteria ilíaca externa, flecha azul arteria ilíaca interna.

Se registró en cada caso: género, lado (derecho o izquierdo), longitud de la arteria ilíaca común (L1), longitud de la arteria iliaca interna (L2), y calibres de la arteria ilíaca común (C1), de la bifurcación ilíaca (C2), de la arteria iliaca interna (C3) y de la arteria ilíaca externa (C4). Los 
hallazgos fueron comparados con las IFU de DRI de tres casas comerciales que se utilizan en nuestro medio: Cook ${ }^{\circledR}$ (Cook Medical, 2017), Jotec ${ }^{\circledR}($ Jotec $\mathrm{GmbH}, 2015)$ y Gore ${ }^{\circledR}$ (Gore et al., 2014).

La medición de los valores antedichos fue hecha con calibre milimetrado y realizada por un único individuo para disminuir así el sesgo del investigador. Los datos fueron registrados en tablas estandarizadas para su posterior análisis. Los datos obtenidos fueron estadísticamente analizados usando SPSS para versión de Windows 23.0. La comparación de variables cuantitativas fue realizada mediante una prueba $t$ para igualdad de medidas. El cruce entre variables cualitativas (sexo y cumplir o no los requisitos para la prótesis, por ejemplo) se realizó mediante el test de chi cuadrado o su corrección de Yates, buscando asociación entre las mismas. Para toda prueba se consideró estadísticamente significativo un valor- $p=<0,05$.

Se realizó una búsqueda electrónica en bases tales como Medline y Pubmed; usando palabras tales como "internal iliac artery", "iliac brach device", "EVAR" en combinación con los operadores booleanos "AND", "OR”. Los artículos recabados se utilizaron para la discusión y análisis de nuestros hallazgos, y son adecuadamente citados al final de esta publicación. Otros trabajos fueron identificados en la bibliografía de los artículos seleccionados inicialmente.

\section{RESULTADOS}

Hemos encontrado una L1 común de promedio 54,52 $\mathrm{mm}$, con un rango de valores entre 33-86 mm y una desviación estándar de 12,22. L2 valor promedio obtenido para esta longitud fue de $40,59 \mathrm{~mm}$, siendo el rango entre $20-77 \mathrm{~mm}$ y el desvío estándar de 10,42. En cuanto a C1, se obtuvo un valor promedio de $11,73 \mathrm{~mm}$ (rango 7$18 \mathrm{~mm}$ y desvío estándar de 2,53). C2 fue de 15,34 mm (rango: 9-23mm y desvío estándar 3,38). El C3 y C4 fue en promedio de $7,28 \mathrm{~mm}$ (rango 4-10mm, desvío estándar: 1,81) y 8,36 $\mathrm{mm}$ (rango 5-15,5 mm, desviación estándar de $2,64)$ respectivamente.

\begin{tabular}{|c|c|c|c|c|c|c|}
\hline Variable & DRI COOK & $\begin{array}{c}\text { Serie actual } \\
\text { n }(\%)\end{array}$ & DRI JOTEC & $\begin{array}{c}\text { Serie actual } \\
\mathrm{n}(\%)\end{array}$ & DRI GORE & $\begin{array}{c}\text { Serie actual } \\
\mathrm{n}(\%)\end{array}$ \\
\hline $\mathrm{L} 1$ & $\begin{array}{l}\text { Mayor o igual } \\
\text { a } 50 \mathrm{~mm}\end{array}$ & $32(60,38 \%)$ & $\begin{array}{l}\text { Mayor o igual } \\
\text { a } 20 \mathrm{~mm}\end{array}$ & $53(100 \%)$ & $\mathrm{NE}$ & $\mathrm{NE}$ \\
\hline $\mathrm{L} 2$ & $\begin{array}{l}\text { Mayor o igual } \\
\text { a } 10 \mathrm{~mm}\end{array}$ & $49(100 \%)$ & $\begin{array}{l}\text { Mayor o igual } \\
\text { a } 15 \mathrm{~mm}\end{array}$ & $49(100 \%)$ & $\begin{array}{l}\text { Mayor a } \\
10 \mathrm{~mm}\end{array}$ & $49(100 \%)$ \\
\hline $\mathrm{C} 2$ & $\begin{array}{l}\text { Mayor o igual } \\
\text { a } 16 \mathrm{~mm}\end{array}$ & $21(42,86 \%)$ & $\begin{array}{l}\text { Mayor o igual } \\
\text { a } 18 \mathrm{~mm}\end{array}$ & $11(22 \%)$ & $\begin{array}{l}\text { Mayor a } \\
14 \mathrm{~mm}\end{array}$ & $28(56 \%)$ \\
\hline $\mathrm{C} 3$ & $\begin{array}{l}\text { Mayor o igual } \\
\text { a } 6 \text { y menor o } \\
\text { igual a } 9 \mathrm{~mm}\end{array}$ & $31(62 \%)$ & NE & NE & $\begin{array}{l}\text { Mayor o igual } \\
\text { a } 6.5 \text { y menor } \\
\text { a } 13.5 \mathrm{~mm}\end{array}$ & $28(56 \%)$ \\
\hline $\mathrm{C} 4$ & $\begin{array}{l}\text { Mayor o igual } \\
\text { a } 8 \text { y menor o } \\
\text { igual a } 11 \mathrm{~mm}\end{array}$ & $20(40 \%)$ & $\begin{array}{l}\text { Mayor o igual } \\
\text { a } 8 \text { y menor o } \\
\text { igual a } 13 \mathrm{~mm}\end{array}$ & $23(46 \%)$ & $\begin{array}{l}\text { Mayor o igual } \\
\text { a } 6.5 \text { y menor } \\
\text { o igual a } \\
25 \mathrm{~mm}\end{array}$ & $37(74 \%)$ \\
\hline $\begin{array}{l}\text { CUMPLEN } \\
\text { TODOS } \\
\text { LOS REQUI- } \\
\text { SITOS }\end{array}$ & & $5(9,43 \%)$ & & $8(15,09 \%)$ & & $18(33,96 \%)$ \\
\hline
\end{tabular}

Tabla 1- Variables en estudio en función de los requisitos de las casas comerciales. Se tabulan las variables en estudio y se comparan según los requisitos de las distintas casas comerciales. En el ítem serie actual se muestran de nuestra serie cuáles casos cumplen con la mencionada característica. Ver texto para referencias.

Al realizar una comparación estadística de las diferencias de longitudes y calibres por sexo, vemos que existieron diferencias significativas en algunos de los valores medidos. La longitud de la arteria ilíaca común mostró una diferencia significativa entre sexos $(p=0,002)$ con una media de $58,14 \mathrm{~mm}$ en el sexo femenino contra $48,05 \mathrm{~mm}$ en el sexo masculino. El calibre de la 
arteria ilíaca común también mostró diferencias significativas $(p=0,001)$, siendo el calibre medio en el sexo femenino de $10,67 \mathrm{~mm}$ mientras que en los preparados masculinos alcanzó los 13,61 $\mathrm{mm}$. En cuanto al calibre en la bifurcación mostró diferencias significativas según sexo $(p=0,003)$; siendo los valores promedios mayores en el sexo masculino que en el femenino: $17,27 \mathrm{~mm}$ y 14,25 $\mathrm{mm}$ respectivamente. Con relación al calibre de la arteria iliaca interna, el calibre medio en el sexo femenino fue de $6,76 \mathrm{~mm}$ y en el masculino de $8,19 \mathrm{~mm}(p=<0,015)$. Por último, el calibre de la arteria ilíaca externa también mostró diferencias significativas $(p=<0,001)$, obteniéndose un calibre medio en el sexo femenino de $7,14 \mathrm{~mm}$ y en el masculino de $10,52 \mathrm{~mm}$. No se halló diferencia significativa entre géneros en la longitud de la arteria iliaca interna.

Luego se procedió a comparar los valores de longitudes y calibres, con los requisitos anatómicos estipulados por las IFU de las casas comerciales que fabrican los DRI, analizando por separado para cada marca comercial. Los principales hallazgos se ilustran en la tabla 1.

Se realizó el cruce de variables entre sexo y compatibilidad con los requisitos para los DRI. Para la casa comercial Cook $\AA$ no se detectó asociación entre el sexo y la aprobación de requisitos, mientras que para las casas comerciales Jotec ${ }^{\circledR}$ y Gore ${ }^{\circledR}$ se halló una asociación estadísticamente significativa entre el sexo masculino y la compatibilidad con los requisitos de los DRI. Los valores $p$ fueron de 0,035 y 0,032 para las casas Jotec y Gore respectivamente.

\section{DISCUSIÓN}

El aneurisma aórtico con aneurisma ilíaco asociado representa un desafío terapéutico para las técnicas de EVAR ya que requieren de extender el sello más allá de la arteria ilíaca común. Como mencionamos existen múltiples técnicas para resolver este problema que podemos resumir en dos grupos: técnicas con preservación de la arteria ilíaca interna y las que no preservan dicho vaso. Dentro de las primeras surgen los DRI que emulan la anatomía del paciente y evitan las complicaciones de ocluir selectivamente la arteria ilíaca interna como ya fue mencionado (Itoga et al, 2017). Algunos de los registros internacionales de utilización de los DRI hablan de un éxito técnico del $85-100 \%$ (Karthikesalingam et al, 2010), una sobrevida a los 12 meses post implante del $98.5 \%$ y una permeabilidad del $92 \%$ de la arteria ilíaca interna (Loth et al, 2015).
Las casas comerciales recomiendan la planificación del procedimiento mediante la utilización de una angiotomografía multicorte (Cook Medical, 2017) en vistas a la evaluación anatómica individualizada del paciente en estudio. En el mismo se deben tomar un conjunto de medidas para determinar si la anatomía del paciente es apta o no para los DRI. Estas mediciones coinciden con las variables: L1, L2, C1, C2, C3 y C4 del presente estudio.

De nuestros resultados podemos observar claras diferencias, estadísticamente significativas, en cuanto a longitudes y calibres en relación con el género; siendo la longitud media de la arteria ilíaca común mayor en los preparados femeninos; mientras que todos los valores de calibre fueron mayores en promedio en los especímenes masculinos. Esto último va en consonancia con otros investigadores que han encontrado menores calibres arteriales en pacientes de género femenino (Krejza et al, 2006). En sintonía con esto, la morfometría de los cadáveres masculinos mostró una diferencia estadísticamente significativa a favor de cumplir con los requisitos de los DRI en comparación con los cadáveres femeninos.

En total, sólo $1(1,89 \%)$ de los preparados cadavéricos estudiados, fue pausible de usar cualquiera de los DRI, ya que sus longitudes y calibres cumplen con cualquiera de las exigencias anatómicas requeridas por cualquiera de las tres casas comerciales. Los valores de calibre y longitud del preparado cadavérico en cuestión fueron de: L1: $67 \mathrm{~mm}$, L2: $45 \mathrm{~mm}$, C2: $20 \mathrm{~mm}$, C3 $9 \mathrm{~mm}$ y C4 $9 \mathrm{~mm}$. Si analizamos de manera combinada los requisitos de las tres casas comerciales, el requisito que generó mayores limitaciones a la a la hora de utilizar los DRI en la población de estudio, fue el diámetro a nivel de la bifurcación ilíaca, donde solo 11 $(20,75 \%)$ preparados se ajustaban a las medidas requeridas.

Si comparamos la idoneidad de las bifurcaciones ilíacas para el uso de DRI, según lo propuesto por Cook $\AA$, en nuestro caso, obtuvimos un $9,43 \%$ de compatibilidad. Pearce B et al. 2015, realizaron una revisión sobre los pacientes que se sometieron a EVAR durante 2004-2013. De estos pacientes, seleccionaron aquellos que tenían aneurismas aorto-ilíacos, 99 casos. Los autores encontraron que el $18,2 \%$ se conformaba con los criterios anatómicos dispuestos.Más de la mitad $(57,6 \%)$ de sus pacientes no elegibles para DRI fueron excluidos por un único criterio anatómico, 23,3\% por dos criterios, y los restantes por tres criterios. A diferencia de nuestro estudio, el principal criterio excluyente fue el diámetro de la arteria iliaca interna. En nuestro caso la exclusión para el DRI de esta 
casa comercial fue: $20,83 \%$ por un único criterio anatómico; un 31,25 \% por dos criterios, 41,66\% por tres criterios y $6,25 \%$ por falta de cuatro criterios. En el mismo artículo (Pearce et al., 2015), los autores compararon también los valores de calibre y longitudes arteriales para los propuestos por la casa comercial Gore B. En dicho caso 25 pacientes de un total de 99 , se ajustaba a los valores preestablecidos (25.3\%). Nuestros resultados por su parte mostraron que 18 (33,96\%) cumplían con los requerimientos. Por su parte, un $38,4 \%$ del total de los excluidos, lo hicieron por la falta de un único criterio, y en nuestro análisis, esto era de un $37,14 \%$.

Otro estudio que valoró el uso de los DRI es el de Itoga et al. 2017. Se estudiaron 87 pacientes procedentes de Japón con aneurismas aortoiliacos. De su grupo en estudio 15 pacientes (17\%) cumplían con los criterios del DRI de Cook (B) y 22 pacientes (25\%) cumplían con todos los requerimientos del DRI de Gore ${ }^{\circledR}$.

Debemos reconocer algunas limitaciones de nuestro estudio. Al tratarse de un estudio cadavérico, los valores de calibre y longitud pueden verse modificados por cambios post mortem así como por la utilización de formaldehído. Otro aspecto a tener presente es la técnica de medición. La mayoría de las casas comerciales sugieren medir "outer-outer" en la tomografía (Jotec $\mathrm{GmbH}$, 2015), lo que significa desde la pared externa a la pared externa contralateral. En el presente estudio, las ramas del cartabón milimetrado se colocan desde la pared externa hacia la contralateral "emulando" a nivel cadavérico una medición "outer-outer" tomográfica. Esto último quizás explique en parte la diferencia de compatibilidad encontrada en nuestro estudio en comparación con los investigadores clínicos.

En conclusión, de nuestros hallazgos y la revisión bibliográfica surge que no todas las bifurcaciones ilíacas cumplen con los requisitos morfométricos para la utilización del DRI. De esto último se desprende que el conocimiento individualizado de la anatomía del paciente en cuestión sea clave para poder determinar la factibilidad del implante de un DRI 0 no. Creemos que se deben doblegar los esfuerzos por lograr mayor adaptabilidad de los DRI a distintas anatomías y que el cirujano conozca las distintas técnicas de sello distal en pacientes con aneurismas aorto-ilíacos ya que como se analizó son pocos los pacientes candidatos al uso de DRI, especialmente si se trata de pacientes de sexo femenino.

\section{Conflicto de intereses}

Los autores declaran no tener conflicto de intereses.

\section{Financiamiento}

Ninguno.

\section{Aprobación ética}

No es necesaria.

\section{Consentimiento informado}

Los cadáveres en los que se realizaron las disecciones, se obtuvieron de donantes voluntarios que expresaron su consentimiento de forma escrita; libre y voluntariamente, en vida. El Departamento de Anatomía de Facultad de Medicina, Universidad de la República, cuenta con dichos consentimientos informados que habilitan a que estos cadáveres sean usados para tareas de docencia e investigación.

\section{Contribuciones}

AMR: Diseño del estudio y elaboración del protocolo, elaboración del manuscrito, aprobación del manuscrito y revisión final. MFI: Diseño del estudio y elaboración del protocolo, disección del material cadavérico y búsqueda bibliográfica, mediciones morfométricas, elaboración del manuscrito y aprobación del manuscrito. LV: Disección del material cadavérico y búsqueda bibliográfica, mediciones morfométricas, elaboración del manuscrito y aprobación del manuscrito. JEK: Procesamiento estadístico y aprobación del manuscrito.

\section{REFERENCIAS}

Armon MP, Wenham PW, Whitaker SC, Gregson $R H$, Hopkinson BR. 1998. Common iliac artery aneurysms in patients with abdominal aortic aneurysms. Eur J Vasc Endovasc Surg 15: 255-57.

Chaikof EL, Dalman RL, Eskandari MK, Jackson BM, Lee WA, Mansour MA, Starnes BW. 2018. The Society for Vascular Surgery practice guidelines on the care of patients with an abdominal aortic aneurysm. J Vasc Surg 67: 277.

Cook Medical. 2017. Physician's pocket reference guide. URL: www.cookmedical.com (consultado en mayo 2020).

Criado FJ, Wilson EP, Velazquez OC, Carpenter $J P$, Barker C, Wellons E, Abul-Khoudoud O, Fairman RM. 2000. Safety of coil embolization of the internal iliac artery in endovascular grafting of abdominal aortic aneurysms. J Vasc Surg 32: 684-88.

D'oria $M$, Mastrorilli $D$, Demartino $R$, Lepidi $S$. 2019. Current status of endovascular preservation of the internal iliac artery with iliac branch devices (IBD). Cardiovasc Intervent 
Radiol 42: 935-48.

Gore WL \& Associates. 2014. Gore excluder iliac branch endoprosthesis - catalogue. URL: www.goremedical.com (consultado en mayo 2020).

Greenhalgh M. 2018. Vascular and endovascular controversies update - 40 year of looking forward. Inglaterra: BIBA Publishing, pág: 1504.

Itoga NK, Fuiimura N, Hayashi $K$, Obara $H$, Shimizu $H$, Lee JT. 2017. Outcomes of endovascular repair of aortoiliac aneurysms and analyses of anatomic suitability for internal iliac artery preserving devices in japanese patients. Circ J 81: 682-88.

Jongsma H, Bekken JA, Bekkers WJJ, Zeebregts CJ, Van Herwaarden J, Hoksbergen A, Cuypers P, de Vries JPPM, Verhagen HJ, Fioole B. 2017. Endovascular treatment of common iliac artery aneurysms with an iliac branch device: Multicenter experience of 140 patients. J Endovasc Ther 24: 239-45.

Jotec GmbH. 2015. E-iliac - product catalogue. URL: www.jotec.com (consultado en mayo 2020).

Karthikesalingam A, Hinchliffe RJ, Holt PJE, Boyle JR, Loftus IM, Thompson MM. 2010. Endovascular aneurysm repair with preservation of the internal iliac artery using the iliac branch graft device. Eur J Vasc Endovasc Surg 39, 285-94.

Kayssi A, Neville RF. 2018. Hypogastric preservation during treatment of aortoiliac aneurysms. Tech Vasc Interv Radiol 21: 17580.

Krejza J, Arkuszewski M, Kasner SE, Weigele J, Ustymowicz A, Hurst RW, Cucchiara BL, Messe $S R$. 2006. Carotid artery diameter in men and women and the relation to body and neck size. Stroke 37: 1103-05.

Loth AG, Rouhani G, Gafoor SA, Sievert $H$, Stelter WJ. 2015. Treatment of iliac artery bifurcation aneurysms with the secondgeneration straight iliac bifurcated device. J Vasc Surg 62: 1168-75.

Oderich GS, Greenberg RK. 2011. Endovascular iliac branch devices for iliac aneurysms. Perspect Vasc Surg Endovasc Ther 23: 16672.

Pearce BJ, Varu VN, Glocker R, Novak Z, Jordan $W D$, Lee JT. 2015. Anatomic suitability of aortoiliac aneurysms for next generation branched systems. Ann Vasc Surg 29: 69-75.

Rayt HS, Bown MJ, Lambert KV, Fishwick NG, McCarthy MJ, London NJM, Sayers RD. 2008. Buttock claudication and erectile dysfunction after internal iliac artery embolization in patients prior to endovascular aortic aneurysm repair. Cardiovasc Intervent Radiol 31: 728-34.

Schneider DB, Matsumura JS, Lee JT, Peterson BG, Chaer RA, Oderich GS. 2017. Prospective, multicenter study of endovascular repair of aortoiliac and iliac aneurysms using the Gore Iliac Branch Endoprosthesis. J Vasc Surg 66: 775-85. 